\title{
Astrometric orbits of spectral binary brown dwarfs - I. Massive T dwarf companions to 2M1059-21 and 2M0805+48
}

\author{
J. Sahlmann ${ }^{\oplus,}, 2 \star$ A. J. Burgasser ${ }^{\odot},{ }^{3}$ D. C. Bardalez Gagliuffi, ${ }^{4}$ P. F. Lazorenko, ${ }^{5}$ \\ D. Ségransan, ${ }^{6}$ M. R. Zapatero Osorio, ${ }^{7}$ C. H. Blake, ${ }^{8}$ C. R. Gelino, ${ }^{9}$ E. L. Martín ${ }^{10,11,12}$ \\ and $\mathrm{H}$. Bouy ${ }^{13}$ \\ ${ }^{1}$ Independent Researcher, 28221 Madrid, Spain \\ ${ }^{2}$ Space Telescope Science Institute, 3700 San Martin Drive, Baltimore, MD 21218, USA \\ ${ }^{3}$ Department of Physics, University of California, San Diego, CA 92093, USA \\ ${ }^{4}$ Department of Astrophysics, American Museum of Natural History, Central Park West at 79th Str, New York, NY 10024, USA \\ ${ }^{5}$ Main Astronomical Observatory, National Academy of Sciences of the Ukraine, Zabolotnogo 27, 03680 Kyiv, Ukraine \\ ${ }^{6}$ Observatoire de l'Université de Genève, Chemin de Pégase 51, 1290 Versoix, Switzerland \\ ${ }^{7}$ Centro de Astrobiología (CSIC-INTA), Carretera Ajalvir km 4, E-28850 Torrejón de Ardoz, Madrid, Spain \\ ${ }^{8}$ Department of Physics and Astronomy, University of Pennsylvania, Philadelphia, PA 19104, USA \\ ${ }^{9}$ NASA Exoplanet Science Institute, Mail Code 100-22, California Institute of Technology, 770 South Wilson Avenue, Pasadena, CA 91125, USA \\ ${ }^{10}$ Instituto de Astrofísica de Canarias (IAC), Calle Vía Láctea s/n, E-38200 La Laguna, Tenerife, Spain \\ ${ }^{11}$ Departamento de Astrofísica, Universidad de La Laguna (ULL), E-38206 La Laguna, Tenerife, Spain \\ ${ }^{12}$ Consejo Superior de Investigaciones Científicas, E-28006 Madrid, Spain \\ ${ }^{13}$ Laboratoire d'astrophysique de Bordeaux, University of Bordeaux, CNRS, B18N, Allée Geoffroy Saint-Hilaire, F-33615 Pessac, France
}

Accepted 2020 April 30. Received 2020 April 28; in original form 2020 January 31

\begin{abstract}
Near-infrared spectroscopic surveys have uncovered a population of short-period, blendedlight spectral binaries composed of low-mass stars and brown dwarfs. These systems are amenable to orbit determination and individual mass measurements via astrometric monitoring. Here, we present first results of a multiyear campaign to obtain high-precision absolute astrometry for spectral binaries using the Gemini-South and Gemini-North GMOS imagers. We measure the complete astrometric orbits for two systems: $2 \mathrm{M} 0805+48$ and 2M1059-21. Our astrometric orbit of $2 \mathrm{M} 0805+48$ is consistent with its 2-yr radial velocity orbit determined previously and we find a mass of $66_{-14}^{+5} M_{\text {Jup }}$ for its T5.5 companion. For 2M1059-21, we find a 1.9-yr orbital period and a mass of $67_{-5}^{+4} M_{\text {Jup }}$ for its T3.5 companion. We demonstrate that sub-milliarcsecond absolute astrometry can be obtained with both GMOS imagers and that this is an efficient avenue for confirming and characterizing ultracool binary systems.
\end{abstract}

Key words: binaries: close - brown dwarfs - astrometry - parallaxes - stars: low-mass.

\section{INTRODUCTION}

The internal and observable properties of normal hydrogen-burning stars can be determined largely from their masses, ages, and compositions (Vogt 1926; Russell 1931). This is not the case for brown dwarfs, low-mass sources incapable of sustained hydrogen fusion (Hayashi \& Nakano 1963; Kumar 1963), which were finally identified in the Pleiades cluster just 25-yr ago (Rebolo, Zapatero Osorio \& Martín 1995). Because these objects cool over time, both mass and age dictate observable properties, challenging the characterization of the local brown dwarf population and substellar mass function (Burgasser 2004; Allen et al. 2005). Disentangling mass and age is a primary motivator for characterizing spectral features sensitive to surface gravity (Allers et al. 2007; Cruz,

^E-mail: josahlmann@gmail.com
Kirkpatrick \& Burgasser 2009; Martín et al. 2010), which are, however, too subtle for sources older than $\sim 200$ Myr (Kirkpatrick et al. 2008; Martin et al. 2017). Other stellar age metrics such as magnetic activity and angular momentum evolution are not useable in the brown dwarf regime (Mohanty \& Basri 2003; Berger 2006; Reiners \& Basri 2008). As a result, we cannot precisely measure the ages or masses of the majority of brown dwarfs in the vicinity of the Sun. In many cases, e.g. for L dwarfs or when the Lithium test (e.g. Magazzu, Martin \& Rebolo 1993) is inconclusive, we cannot even determine if they are brown dwarfs or stars, although recent progress is being made by extending the determination of the lithium boundary method $\mathrm{L}$ dwarf members in clusters older than it was previously thought to be possible, such as the Hyades (e.g. Martín et al. 2018).

Over the past decade, mass measurements have been achieved for dozens of low-mass stellar and sub-stellar binaries in the field (cf. Dupuy \& Liu 2017). These are primarily resolved systems, 
but there have also been a smaller number of mass measurements from radial velocity (RV; Basri \& Martín 1999; Guenther \& Wuchterl 2003; Joergens \& Müller 2007; Blake et al. 2008; Blake, Charbonneau \& White 2010; Joergens, Müller \& Reffert 2010; Burgasser et al. 2010, 2012b, 2016a; Konopacky et al. 2010) and astrometric orbits (Sahlmann et al. 2015, 2013; Koren et al. 2016), as well as microlensing masses (e.g. Bennett et al. 2008; Han et al. 2013; Poleski et al. 2017; Miyazaki et al. 2018). The direct measurement of both spectra and masses for resolved short-period binaries have permitted tests of evolutionary models, revealing in many cases systematic discrepancies (e.g. Konopacky et al. 2010; Dupuy, Liu \& Ireland 2014; Dieterich et al. 2018; Brandt et al. 2019). Yet the number of resolvable systems (angular separation $\gtrsim 50$ mas equivalent to $\gtrsim 1$ au at $25 \mathrm{pc}$ ) with short enough orbital periods for mass measurement $(P \lesssim 10 \mathrm{yr})$ has reached a limit until the resolving power of $30 \mathrm{~m}$ telescopes becomes available. Other methods yield fewer binaries and less information: radial-velocity (RV) monitoring ( $\lesssim 10$ per cent yield) probes shorter orbits but provide only mass limits because of the unknown orbit inclination and little information on secondary atmospheres. Astrometric variables are rarer ( $\lesssim 5$ per cent yield) and provide limited information on the secondary spectrum. Microlenses offer precise masses but no atmospheric information whatsoever.

Fortunately, close-separation, unequal-mass binaries straddling the hydrogen-burning limit can be identified as blended-light spectral binaries (Cruz et al. 2004; Burgasser et al. 2008, 2010; Bardalez Gagliuffi et al. 2014). These systems are composed of a late-M or L dwarf primary and a T dwarf secondary, which have distinct spectral morphologies (Kirkpatrick 2005). They are efficiently revealed through peculiar features in low-resolution, near-infrared spectra, which can also be used to characterize the atmospheres of the binary components. Their separation-independent identification permits the detection and orbital measurement of potentially short-period systems. Over 60 spectral binary candidates have been identified to date, and roughly a dozen confirmed through high-resolution imaging, RV monitoring, and astrometric monitoring (Bardalez Gagliuffi, Gelino \& Burgasser 2015 and references therein). These include some of the most tightly separated very low-mass binaries known, for which both orbit and mass measurements have been achieved, primarily through RV monitoring ( $<1$ au; Blake et al. 2008; Burgasser et al. 2008, 2012a, 2016a).

However, RV monitoring typically provides only one axis of the primary's orbital motion. Fortunately, the proximity and extreme flux ratios of very low-mass spectral binaries make them ideal for the measurement of astrometric variability and therefore all orbital parameters (e.g. Brandner et al. 2004; Dahn et al. 2008; Dupuy \& Liu 2012; Sahlmann et al. 2013). Here, we report first results from a long-term, ground-based astrometric follow-up survey targeting very low-mass spectral binaries.

\section{ASTROMETRIC BINARY SURVEY SAMPLE}

The amplitude of astrometric variability, i.e. the size of a binary's photocentre orbit $\alpha=a_{\text {rel }}(f-\beta)$, depends on the projected angular semimajor axis of the orbit $a_{\text {rel }}$, the fractional mass $f=$ $M_{2} /\left(M_{1}+M_{2}\right)$ and the fractional flux $\beta=F_{2} /\left(F_{1}+F_{2}\right)=$ $\left(1+10^{0.4 \Delta m}\right)^{-1}$, where $\Delta m$ is the magnitude difference between the components in a given photometric band. Very low-mass spectral binaries, particularly those with late-M and early-L primaries, can have very large optical flux ratios $\left(F_{2} / F_{1} \ll 1 \Rightarrow \beta \ll 1\right)$ and modest mass ratios $\left(q=M_{2} / M_{1} \simeq 0.5-0.8 \Rightarrow f \simeq 0.5-0.7\right)$, depending on the system age (Burgasser \& Blake 2009). Hence, spectral binaries should in principle exhibit non-zero astrometric perturbations. Detectable astrometric variables must strike a balance between large-amplitude but long-period systems that could be resolved by direct imaging ( $\alpha \gtrsim 20-50$ mas for $a_{\text {rel }}>50-100$ mas; $P \gtrsim 3-8 \mathrm{yr}$ ); and short-period but small-amplitude systems that could be identified by RV monitoring ( $\alpha \lesssim 3-5$ mas for $a_{\text {rel }} \lesssim$ 0.3 au at $25 \mathrm{pc} ; P \lesssim 0.5 \mathrm{yr}$, RV signature $\left.\lesssim 5-10 \mathrm{~km} \mathrm{~s}^{-1}\right)$. Hence, our optimal targets should be unresolved by direct imaging, and may or may not show some evidence of RV variability, encompassing periods of $0.5 \mathrm{yr} \lesssim P \lesssim 3 \mathrm{yr}$.

Starting from the 60 spectral binaries compiled by Bardalez Gagliuffi et al. (2014) and subsequent discoveries, we selected sources within $40 \mathrm{pc}$ for which high-resolution images were unresolved, and for which the inferred $\Delta J>1$ (based on modelling of the blended spectrum), implying $\Delta I \gtrsim 4$ based on the $I-$ $J /$ spectral type relation of Hawley et al. (2002). We also excluded sources with $I>22$. We prioritized sources for which RV variations are evident in follow-up Keck/NIRSPEC monitoring (Burgasser et al. 2016a; Burgasser et al. in preparation). The final sample consists of 10 spectral binaries and binary candidates, listed in Table 1.

These targets were observed in three long-term astrometric monitoring campaigns. Eight sources (four in the north and four in the south) were monitored with the Gemini Multi-Object Spectrograph (GMOS; Hook et al. 2004; Gimeno et al. 2016) on the Gemini-North ${ }^{1}$ and - South $^{2}$ telescopes (PI: Burgasser). Two sources (SDSS J0931+28 and 2MASS J1453+14) were observed with OSIRIS (Cepa et al. 2000) on the Gran Telescopio Canaria (GTC; PI: Sahlmann). In this paper, we focus on the results of our GMOS observations of $2 \mathrm{M} 0805+48$ and 2M1059-21; the remaining targets will be discussed in subsequent publications.

2M1059-21: This source has an L1 near-infrared, combinedlight classification (Cruz et al. 2003), yet it was identified as a candidate spectral binary of L1+T3 dwarf components (Bardalez Gagliuffi et al. 2014).

2M0805+48: This source was first identified as an unusually blue L dwarf with discrepant optical and near-infrared classifications of L4 and L9 (Hawley et al. 2002 and Knapp et al. 2004, respectively). Burgasser (2007) posited that its unusual nearinfrared spectrum could be due to the combined light of a L4.5 and T5 dwarf components. Dupuy \& Liu (2012) confirmed this source as an astrometric variable with an amplitude of $\sim 15$ mas, and estimated a period of 2.7-9.1 yr and a semimajor axis of 0.9-2.3 au. They also inferred a similar spectral component composition of L4+T5. High-resolution laser guide-star adaptive-optics imaging observations with Keck were unable to resolve this system (Bardalez Gagliuffi et al. 2015), setting a separation upper limit of 170 mas. Burgasser et al. (2016a) monitored this system with high-resolution infrared spectroscopy and detected significant RV variability over the course of $4 \mathrm{yr}$. Their orbit fits yielded a period of $2.02 \pm 0.03 \mathrm{yr}$, a semimajor axis of $0.76_{-0.06}^{+0.05} \mathrm{au}$, and a non-zero eccenticity of $0.46 \pm 0.05$. By combining their measurements with brown dwarf evolutionary models, Burgasser et al. (2016a) also deduced that the system was close to edge-on $\left(90^{\circ} \pm 19^{\circ}\right)$ and has a large system mass ratio $\left(q=0.86_{-0.12}^{+0.10}\right)$, substellar-mass components

\footnotetext{
${ }^{1}$ Programmes GN-2017A-Q-24, GN-2017B-Q-4, GN-2018A-Q-128, GN2018B-Q-104, GN-2019A-Q-231, GN-2019B-Q-104

${ }^{2}$ Programmes GS-2015A-Q-69, GS-2015B-Q-2, GS-2016B-Q-36, GS2017A-Q-53, GS-2017B-Q-6, GS-2018A-Q-133
} 
Table 1. Target sample.

\begin{tabular}{|c|c|c|c|c|c|c|c|c|}
\hline Name & Identifier & $\begin{array}{c}\text { Combined } \\
\text { type }\end{array}$ & $\begin{array}{c}\text { Component } \\
\text { types }\end{array}$ & $\begin{array}{c}I \\
(\mathrm{mag})\end{array}$ & $\begin{array}{c}\Delta J \\
(\mathrm{mag})\end{array}$ & $\begin{array}{c}\Delta I \\
(\mathrm{mag})\end{array}$ & Instrument & Ref \\
\hline $2 \mathrm{M} 0805+48$ & SDSS J080531.84+481233.0 & L4/L9 & $\mathrm{L} 4+\mathrm{T} 5.5$ & $19.59 \pm 0.03$ & 1.5 & 3.8 & Gemini-N/GMOS & $1-4$ \\
\hline $2 \mathrm{M} 1711+22$ & 2MASSI J1711457+223204 & L6.5/L9.5 & $\mathrm{L} 5+\mathrm{T} 5.5$ & $21.92 \pm 0.15$ & 0.9 & 3.3 & Gemini-N/GMOS & 2,5 \\
\hline $2 \mathrm{M} 2126+76$ & 2MASS J21265916+7617440 & T0p & $\mathrm{L} 8.5+\mathrm{T} 4.5$ & $20.13 \pm 0.03$ & 0.4 & 1.9 & Gemini-N/GMOS & 6 \\
\hline WI0720-08 & WISE J072003.20-084651.2 & M9.5 & $\mathrm{M} 9.5+\mathrm{T} 5$ & $14.93 \pm 0.02$ & 2.6 & 5.5 & Gemini-S/GMOS & $7-9$ \\
\hline 2M2026-29 & 2MASS J20261584-2943124 & $\mathrm{L} 1$ & $\mathrm{~L} 0+\mathrm{T} 6$ & $19.13 \pm 0.02$ & 3.4 & 6.5 & Gemini-S/GMOS & 5,10 \\
\hline SD0931+28 & SDSS J093113.23+280227.1 & L3 & $\mathrm{L} 1.5+\mathrm{T} 2.5$ & $19.44 \pm 0.03$ & 2.2 & 4.4 & GTC/OSIRIS & 5 \\
\hline $2 \mathrm{M} 1453+14$ & 2MASS J14532582+1420410 & L1 & $\mathrm{L} 1+\mathrm{T} 6$ & $19.65 \pm 0.02$ & 3.3 & 6.2 & GTC/OSIRIS & 5 \\
\hline
\end{tabular}

Notes. (1) Burgasser (2007); (2) Burgasser et al. (2010); (3) Dupuy \& Liu (2012); (4) Burgasser et al. (2016a); (5) Bardalez Gagliuffi et al. (2014); (6) Bardalez Gagliuffi et al. (2015); (7) Burgasser et al. (2015a); (8) Burgasser et al. (2015b); (9) Dupuy et al. (2019); (10) Gelino \& Burgasser (2010).

Table 2. Gemini data analysed in this paper. The individual frame exposure time (in seconds), typical number of dithers per epoch $N_{\mathrm{d}}$, number of epochs $N_{\mathrm{e}}$, total number of frames $N_{\mathrm{f}}$, and number of available reference stars $N_{\star}$ are listed, as well as the total time span covered by the observations (in days)

\begin{tabular}{lcccccc}
\hline Source & $\begin{array}{c}\text { Exp. } \\
(\mathrm{s})\end{array}$ & $N_{\mathrm{d}}$ & $N_{\mathrm{e}}$ & $N_{\mathrm{f}}$ & $\begin{array}{c}\text { Time span } \\
(\mathrm{d})\end{array}$ & $N_{\star}$ \\
\hline 2M0805+48 & 200 & 10 & 13 & 125 & 786 & 67 \\
2M1059-21 & 180 & 12 & 19 & 258 & 1153 & 51 \\
\hline
\end{tabular}

$\left(M_{1}=0.057_{-0.014}^{+0.016} \mathrm{M}_{\odot}, M_{2}=0.048_{-0.010}^{+0.008} \mathrm{M}_{\odot}\right)$, and a relatively old age ( $\tau \gtrsim 4$ Gyr), although these values are highly model dependent.

\section{OBSERVATIONS}

\subsection{Gemini GMOS imaging astrometry}

Our programme is modelled on the astrometric survey of ultracool dwarfs described in Sahlmann et al. (2013), Sahlmann et al. (2014), which demonstrated 0.1 mas accuracy for M8-L2.5 dwarfs down to $I=17.5$ using the VLT/FORS2 instrument (126 mas pixel ${ }^{-1}$ ) and the method of Lazorenko et al. (2014). We conducted a pilot programme in 2015 to test the application of this method with Gemini-S/GMOS, whose Hamamatsu detector has comparable field of view, more red sensitivity, and a smaller pixel scale (80 mas) than VLT/FORS2. Upon verifying that comparable astrometric precision could be achieved, we conducted monitoring programmes with Gemini-S from 2016 to 2018 and with Gemini-N (after the installation of the Hamamatsu detector for GMOS-N) from 2017 to 2019. Table 2 summarizes the data analysed here.

The observation design was straightforward. Multiple epochs of imaging separated by roughly one month during visibility periods were obtained over several years. Each epoch consisted of a sequence of $8-12$ dithered (1 arcsec random dither pattern) exposures in the $i$-band filter, with exposure times designed to yield $\mathrm{S} / \mathrm{N}>50$ per exposure. The field-of-view centring and orientation were maintained to be as constant as possible throughout the monitoring period, and observations were obtained at small airmass $(<1.5)$ and close to meridian to reduce differential colour refraction (DCR). Reasonable imaging conditions $(<0.75$ arcsec) and sky transparency (cirrus only or better) were also required. All observations were requested and executed in queue mode.
Table 3. Additional Keck NIRSPEC radial velocity measurements of $2 \mathrm{M} 0805+48$ and $2 \mathrm{M} 1059-21$.

\begin{tabular}{lcccc}
\hline Date (UT) & MJD & S/N & $\begin{array}{c}\mathrm{RV} \\
\left(\mathrm{km} \mathrm{s}^{-1}\right)\end{array}$ & $\begin{array}{c}v \sin i \\
\left(\mathrm{~km} \mathrm{~s}^{-1}\right)\end{array}$ \\
\hline 2M0805+48 & - & - & - & - \\
2017 Mar 22 & 57834.35930 & 19 & $+7.7 \pm 0.5$ & $36.4 \pm 0.8$ \\
2017 Dec 7 & 58094.63541 & 11 & $+14.1 \pm 0.8$ & $35.4 \pm 1.2$ \\
2018 Jan 1 & 58119.49976 & 4 & $+17.3 \pm 1.1$ & $33.9 \pm 2.4$ \\
2M1059-21 & - & - & - & - \\
2016 Apr 22 & 57500.30332 & 18 & $+40.0 \pm 0.3$ & $13 \pm 2.2$ \\
2016 May 22 & 57530.26493 & 16 & $+40.0 \pm 0.3$ & $14.6 \pm 1.6$ \\
\hline
\end{tabular}

\subsection{Keck NIRSPEC radial velocities}

We obtained new high-resolution spectroscopy of 2M0805+48 and 2M1059-21 with Keck/NIRSPEC (McLean et al. 1998) at multiple epochs. The data acquisition, reduction, and RV determination were performed as described in e.g. Burgasser et al. (2012a) and Burgasser et al. (2016a). The resulting measurements are listed in Table 3, and add to the existing measurements for $2 \mathrm{M} 0805+48$ reported in Burgasser et al. (2016a).

\section{ASTROMETRIC DATA REDUCTION AND ANALYSIS}

\subsection{Basic data reduction}

We used the Gemini data reduction package ${ }^{3}$ to perform the bias and flat-field corrections of the GMOS images. The identification of applicable calibration data and the generation of master bias and master flat files were automated using PYTHON scripts that interface with the Gemini Archive (Sahlmann 2019a). ${ }^{4}$

\subsection{Source extraction and astrometric analysis}

We used Source Extractor (Bertin \& Arnouts 1996) and PSF Extractor (Bertin 2006) to identify sources in every image frame and determine their pixel positions. After a preliminary source extraction, the PSF Extractor tool was run to generate an empirical PSF with parameters that vary across the field. Then, Source

\footnotetext{
${ }^{3} \mathrm{https}: / /$ www.gemini.edu/sciops/data-and-results/processing-software

${ }^{4}$ https://github.com/Johannes-Sahlmann/gemini-reduction
} 
Table 4. Reference frames used for absolute astrometric alignment. The reference frame corresponds to the archive file name which encodes the date and the sequence number, $k$ indicates the degree $d=k / 2-1$ of the polynomial fit (Lazorenko \& Lazorenko 2004), $r_{\mathrm{x}}$ is the initial cross-match radius, $N_{\mathrm{Gaia}}$ is the number of high-fidelity GDR2 sources within 4 arcmin of the target, $N_{\text {image }}$ is the number of extracted sources in the reference frame, $N_{\mathrm{x}}$ is the number of cross-matched sources used for the absolute alignment, and the last four columns list the pixel scales at the reference point and their fit residuals in both dimensions.

\begin{tabular}{|c|c|c|c|c|c|c|c|c|c|c|c|}
\hline Object & Instrument & Reference frame & $k$ & $\begin{array}{c}r_{\mathrm{X}} \\
(\operatorname{arcsec})\end{array}$ & $N_{\text {Gaia }}$ & $N_{\text {image }}$ & $N_{\mathrm{x}}$ & $\begin{array}{l}\text { Scale } x \\
\text { (mas) }\end{array}$ & $\begin{array}{l}\text { Scale } y \\
\text { (mas) }\end{array}$ & $\begin{array}{l}\operatorname{rms} x \\
\text { (mas) }\end{array}$ & $\begin{array}{l}\mathrm{rms} y \\
\text { (mas) }\end{array}$ \\
\hline $2 \mathrm{M} 0805+48$ & GMOS-N & N20171102S0357 & 10 & 4.0 & 49 & 177 & 22 & $80.909 \pm 0.033$ & $80.937 \pm 0.020$ & 1.3 & 1.2 \\
\hline
\end{tabular}

Extractor was provided with the PSF model and performs the final extraction including the pixel position determination. The inter-frame identification of sources was performed using SCAMP (Bertin 2006), however, the astrometric information provided by that tool was not used.

\subsection{Absolute astrometry in the Gaia reference frame}

We based the absolute astrometric calibration of our images on the second Gaia data release (GDR2, Gaia Collaboration et al. 2018, 2016). We accessed the Gaia archive using pygacs ${ }^{5}$ and downloaded sources from the gaiadr2.gaia_source table within a 4 arcmin radius of the target. To increase the fidelity of Gaia sources, we selected only entries with astrometric_excess_noise $<2$ mas, duplicated_source $=0$, ra_error $<3$ mas and dec_error $<3$ mas. We then computed the source positions and their uncertainties at the reference epoch, whose determination is described below. We took into account the five parameters of the standard astrometric model (positions, parallax, and proper motions) and their covariances when applicable.

To identify the reference frame for absolute alignment, we crossmatched the GDR2 sources with the sources extracted in every frame. The cross-match includes an iterative approach in which a two-dimensional distortion model (a bivariate polynomial of degree 4 ) is fit at every iteration and used to continuously refine the position of extracted sources, implemented in pystortion (Sahlmann, Lim \& Noss 2019). ${ }^{6}$ We chose the reference epoch as the image sequence with a large number of cross-matched sources and stable distortion parameters. Within the reference epoch, we chose the reference frame as the one having the largest number of crossmatched sources.

Finally, we performed the absolute astrometric calibration of the reference frame by fitting a two-dimensional distortion model between the GDR2 source positions that were tangent-plane projected with a reference point located at the centre of the field, and the pixel positions of extracted sources. This reference frame defines the absolute coordinates of the measured sources in two ways: it determines the camera distortion and it ties the pixel-based coordinates to absolute stellar coordinates. Table 4 lists the reference frame characteristics for the two targets.

\subsection{Correction of atmospheric image motion and variable distortion}

After application of the absolute astrometric calibration of the reference frame on to the International Celestial Reference Frame

\footnotetext{
${ }^{5}$ https://github.com/Johannes-Sahlmann/pygacs

${ }^{6}$ Using https://github.com/spacetelescope/pystortion
}

(ICRF) realized by Gaia DR2, we determined the 2D polynomial transformation between every frame and the reference frame. In contrast to the absolute calibration, we can use all extracted stars in the image frames (in practice, some sources are discarded as part of the process as described below). We applied the methods of Lazorenko et al. (2014) and Lazorenko et al. (2009) in a slightly simplified fashion.

First, we applied the polynomial transform that was determined for the absolute alignment of the reference frame to all frames of each target field. This step transforms pixel positions into tangent-plane projected angular coordinates that are still affected by changes in the telescope pointing/orientation, variable distortion, and atmospheric image motion.

Secondly, we determined the 2D polynomial transformation between each frame and the reference frame for a given target. These transformations correct for the three effects mentioned above, and yield absolute astrometry in every frame for all measured sources. The target is excluded in this step because it is not used to define the astrometric reference field; instead, the target's motion is determined relative to the astrometric frame defined by the reference stars. In Fig. 1, we show the evolution of the six firstorder distortion parameters (offsets, scale, rotation, and skew terms) in the 2M1059-21 field, for which 50 reference stars passed all the iterative filter stages and a fourth-degree bivariate polynomial was used to map every frame to the reference frame. The residual root mean square (rms) of the fits was typically 5-10 mas for both axes. Changes in the telescope's reported position angle can be seen in the rotation term, and scale and skew changes with amplitudes $^{7}$ up to $7 \times 10^{-4}$ were measured. The sudden scale change around frame 150 corresponds to the time gap between the 2017 and 2018 seasons. The scale difference between the two axes of the GMOS-S detector exhibits a steady increase, whereas the non-perpendicularity between the axes appears to stabilize over time.

The third step consisted of fitting the per-epoch astrometry of individual sources with the appropriate model. The astrometric measurements of the target are $\alpha_{m}^{\star}=\alpha \cos \delta$ and $\delta_{m}$, corresponding to Right Ascension and Declination, respectively, in frame $m$ at time $t_{m}$ relative to the reference frame of background stars. These are modelled with six free parameters $\Delta \alpha_{0}^{\star}, \Delta \delta_{0}, \mu_{\alpha^{\star}}, \mu_{\delta}, \varpi$, and $\rho$ as

$\alpha_{m}^{\star}=\Delta \alpha_{0}^{\star}+\mu_{\alpha^{\star}} t_{m}+\varpi \Pi_{\alpha, m}-\rho f_{1, x, m}$,

$\delta_{m}=\Delta \delta_{0}+\mu_{\delta} t_{m}+\varpi \Pi_{\delta, m}+\rho f_{1, y, m}$,

${ }^{7}$ This value are unitless because all positions are measured in arcseconds relative to the reference position. 

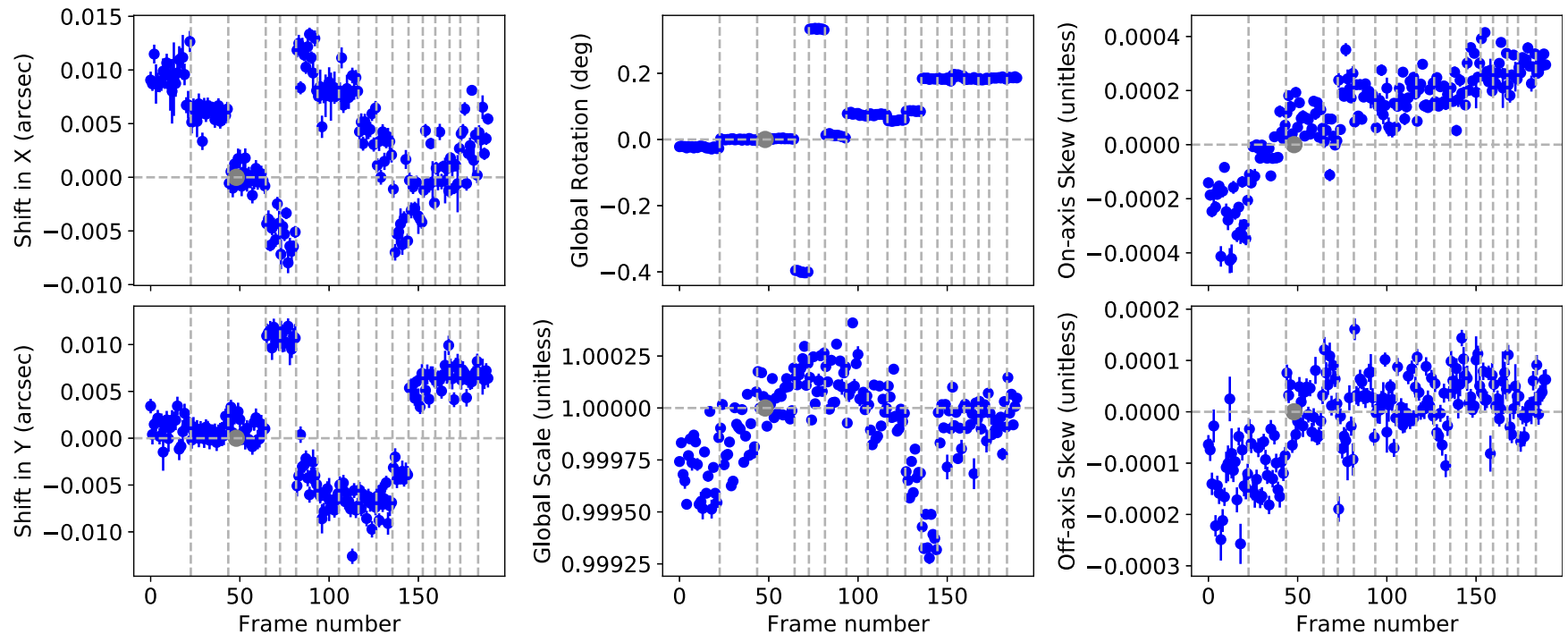

Figure 1. Evolution of inter-frame distortion parameters in the 2M1059-21 field as the result of the iterative reduction described in Section 4.4. Blue symbols show the frame-by-frame evolution relative to the reference frame indicated by the larger grey circle, which is tied to the Gaia system. Vertical dashed lines indicate the epoch boundaries. Left: Residual lateral offsets (these are not the telescope pointing errors because these parameters are being minimized by the procedure). Middle: Global rotation and scale. The rotation figure shows the variation in position angle relative to the reference frame. Right: On-axis skew (i.e. the scale difference between the axes) and the off-axis skew (i.e. the non-perpendicularity between the axes).

where $\Delta \alpha_{0}^{\star}, \Delta \delta_{0}$ are the coordinate offsets, $\mu_{\alpha^{\star}}, \mu_{\delta}$ are the proper motions, and the parallactic motion is expressed as the product of relative parallax $\varpi$ and the parallax factors $\Pi_{\alpha}, \Pi_{\delta}$. The atmospheric refraction modelled by $\rho$ in equation (1) has one parameter less than the model used for our FORS2 work (Lazorenko et al. 2011; Sahlmann et al. 2014) because neither Gemini-South nor Gemini-North incorporates a dispersion compensator. In this case, the differential chromatic refraction (DCR) is modelled with the free parameter $\rho$ and the coefficient $f_{1}$, where the latter is fully determined as a function of zenith angle, temperature, and pressure (Lazorenko 2006; Sahlmann et al. 2013, 2016a). The DCR treatment does not involve the estimation of source colours, instead $\rho$ is an empirical free model parameter that corresponds to the effective colour of the target relative to the average reference star.

This reduction technique is applicable equally both to the target and any field star with its unique set of reference stars. Astrometric solutions for field stars were used to test the presence of systematic errors, to derive the parallaxes of background stars, to corrected to absolute parallax, to determine the pixel scale, and to compile the catalogue of field stars.

As discussed in Lazorenko et al. (2009, section 3.3), the final solution of this third step must be found by iterating steps two and three while imposing an additional set of constraints on the astrometric parameters of the reference stars (see also section 4.1 of Sahlmann 2012). Essentially, this procedure allows us to determine the astrometric parameters (e.g. relative parallax and proper motion) of reference stars while also correcting for the associated epochdependent displacements from a rigid reference field against which the target's motion can be measured. The primary constraint imposed on this iterative process is that the sum of astrometric parameters across reference stars vanishes; i.e. that the sum of their relative parallaxes is zero.

Fig. 2 shows the evolution of the parallax and proper motion parameters for 2M1059-21 as a function of iteration number. It demonstrates the significant benefit of using this approach and the need for 15-20 iterations to allow the algorithm to converge.

\subsection{Correction to absolute parallax and proper motion}

To correct the relative astrometric parameters to absolute quantities, we cross-matched the reference stars with Gaia DR2 sources for which Gaia determined parallaxes. The differences in parallax and proper motions are combined with weights corresponding to their respective uncertainties to obtain the corrections from our relative proper motions and parallaxes to the GDR2 system. Fig. 3 shows these comparisons in the 2M1059-21 field and Table 5 shows the derived corrections (see also the discussion in Section 6.3).

\subsection{Fitting the standard model of parallaxes and proper motions}

In all of the following analyses, we are using all the individual frame data for the model fitting. For better visualization of the results, however, we display only the epoch averages in the figures.

Upon convergence of the iterative astrometric fitting procedure, we adjusted the standard linear model equation (1) to the relative positions of the target in the reference field. Figs 4 and 5 show the results of fitting this six-parameter model to the astrometry data. Very large excess residual noise is detected for both sources, as expected for the expected binary astrometric motion of our targets.

\subsection{Modelling the target's orbital photocentre motion and radial velocity}

The Keplerian orbit model adds an additional seven free parameters to the relative offsets $\Delta \alpha^{\star}$ and $\Delta \delta$ of the target's position. These are the eccentricity $e$, the argument of periastron $\omega$, the orbital period $P$, the longitude of ascending node $\Omega$, the orbital inclination $i$, the time of periastron passage $T_{\mathrm{P}}$, and the semimajor axis of the photocentre orbit $\alpha$. We also include the astrometric nuisance offset parameters $s_{\alpha}$ and $s_{\delta}$ (Sahlmann et al. 2013).

For the spectral binaries in our sample, we estimated the magnitude difference between the components in the filter bandpass, which allows us to relate the photocentre orbit size to the barycentre 

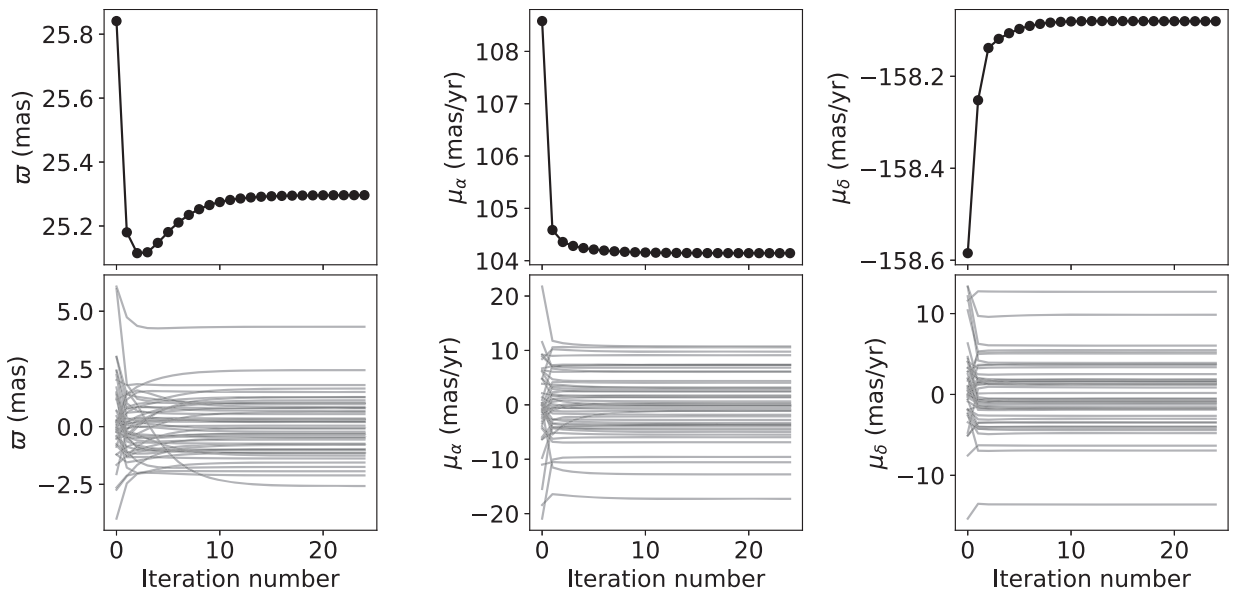

Figure 2. Effects of the iterative analysis for 2M1059-21. The evolution of the best-fitting parallax (left), proper motion in RA (middle), and Dec. (right) is shown as a function of iteration number for the target (top row) and the reference stars (bottom row).
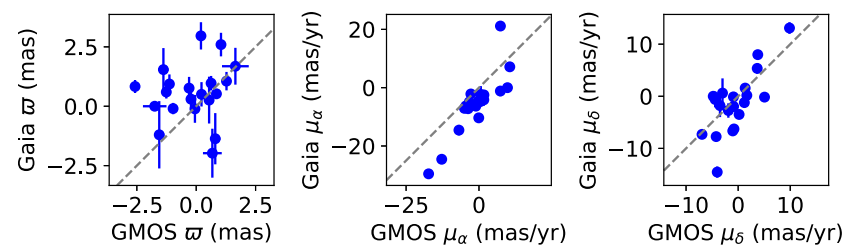

Figure 3. Comparison of parallaxes and proper motions between our determinations and GDR2 in the 2M1059-21 field. In this case, there are 21 reference stars that are both in GDR2 and are used in the final iteration. These are used to derive the corrections to absolute.

Table 5. Corrections to absolute parallax and proper motion. These offsets need to be added to the relative parameters. The number of used reference stars is $N_{\text {ref. }}$.

\begin{tabular}{lcccc}
\hline Object & $N_{\text {ref }}$ & $\begin{array}{c}\Delta \varpi \\
(\mathrm{mas})\end{array}$ & $\begin{array}{c}\Delta \mu_{\alpha^{\star}} \\
\left(\mathrm{mas} \mathrm{yr}^{-1}\right)\end{array}$ & \multicolumn{1}{c}{$\begin{array}{c}\Delta \mu_{\delta} \\
\left(\mathrm{mas} \mathrm{yr}^{-1}\right)\end{array}$} \\
\hline 2M1059-21 & 21 & $0.82 \pm 0.11$ & $-5.64 \pm 0.52$ & $-0.12 \pm 0.39$ \\
2M0805+48 & 9 & $0.53 \pm 0.11$ & $1.48 \pm 0.77$ & $-0.75 \pm 0.35$ \\
\hline
\end{tabular}

orbit size $a_{1}$ of the primary, as described in Section 2. The relative $i$-band magnitude differences (Table 1) were inferred from the relative 2MASS $J$-band magnitude differences estimated from spectral decomposition analysis (Bardalez Gagliuffi et al. 2014; Burgasser et al. 2016) and component $i-J$ colours based on the empirical colour/spectral-type relations of Skrzypek, Warren \& Faherty (2016). Uncertainties in the colour relation, component spectral types, and relative $J$ magnitudes (which dominated the error budget) were propagated to compute the relative $i$-band magnitude difference uncertainty. In addition, we established prior estimates for the primary mass $M_{1}$ from a population synthesis simulation. We generated $10^{4}$ simulated ultracool dwarfs assuming a constant age distribution, a mass distribution $\frac{\mathrm{d} N}{\mathrm{~d} M} \propto M^{-0.5}$ over $0.01 \leq$ $\mathrm{M} \leq 0.15$, evolutionary models from Martín et al. (2003), and an empirical temperature/spectral-type mapping from Pecaut \& Mamajek (2013). We sampled the masses of all sources with simulated spectral types within \pm 0.5 subtypes of the inferred primary component. Prior knowledge on $M_{1}$ allows us to directly fit the companion mass $M_{2}$ instead of $\alpha$.

The resulting primary mass distribution for 2M1059-21 is shown in Fig. 6. We approximated this distribution with the empirical

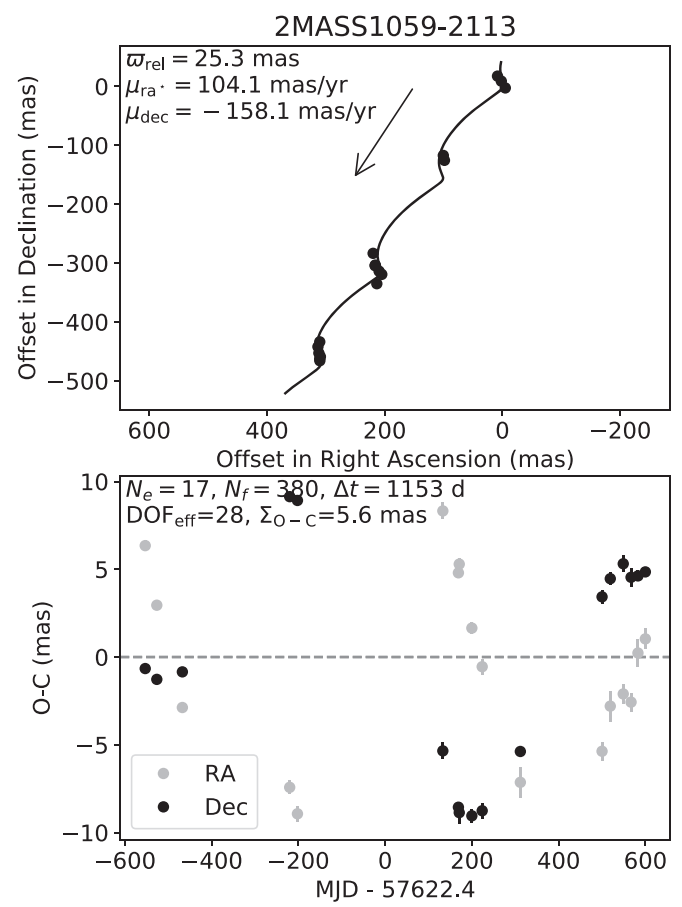

Figure 4. Results of fitting the six-parameter model (PPM+DCR) to 2M1059-21. The on-sky motion (top) and the epoch residuals (bottom) are shown. Significant excess signal is evident.

probability distribution function (dotted blue line), which we used to implement a primary mass prior for the MCMC. The resulting posterior distribution is shown with a solid black line and reproduces the input samples well. We implemented the same process for 2M0805+48.

To mitigate the effects of correlations and limitations that naturally exists for certain orbital parameters, we transform between the following combinations as needed: $\lambda_{\text {Ref }} \Longleftrightarrow T_{\mathrm{P}}$, where $\lambda_{\text {Ref }}$ is the mean longitude at time $T_{\text {Ref }} ;\left(M_{2}, i\right) \Longleftrightarrow\left(\sqrt{M_{2}} \sin i, \sqrt{M_{2}} \cos i\right)$; and $(e, \omega) \Longleftrightarrow(\sqrt{e} \sin \omega, \sqrt{e} \cos \omega)$

The radial velocity of the binary is fully characterized by the parameters above with the addition of the systemic velocity $\gamma$. 

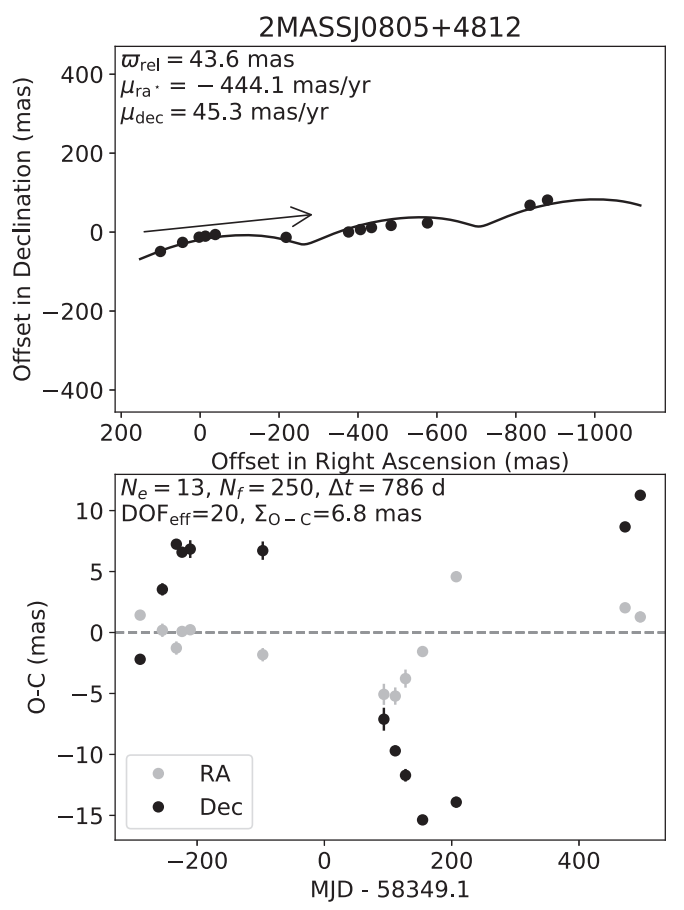

Figure 5. Results of fitting the six-parameter model (PPM+DCR) to $2 \mathrm{M} 0805+48$.

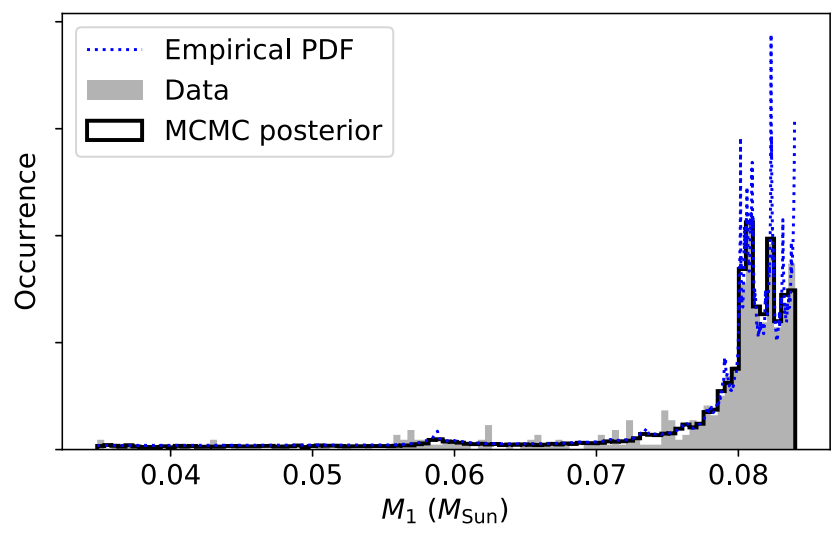

Figure 6. Primary mass distribution used for 2M1059-21.

\subsection{Search for and characterization of orbital motion}

Since the standard model for astrometric motion does not fit the data well, we searched for orbital motion for all targets using the methods described in Sahlmann et al. (2013). A Genetic Algorithm is employed to efficiently sample the allowed ranges of non-linear parameters $\left(P, e, T_{P}\right)$ and as the algorithm evolves the regions of minimum $\chi^{2}$ are determined.

An inherent parameter degeneracy exists when determining orbital parameters from astrometric data, in that the two solutions with $(\omega, \Omega)$ and $\left(\omega+180^{\circ}, \Omega+180^{\circ}\right)$ are indistinguishable. We will call the second solution the 'degenerate' orbit. The two can be disentangled when RV data are available because the RV signature of the degenerate orbit is inverted about the systemic velocity, i.e. $\mathrm{RV}_{\text {degenerate }}=-\mathrm{RV}_{\text {nominal }}+\gamma$.

The best-fitting parameters determined by the Genetic Algorithm were used as starting values for a Markov Chain Monte Carlo (MCMC) analysis similar to that described in Sahlmann et al.

Table 6. MCMC posterior parameters of 2M1059-21. The parameter $M_{\text {tot }}$ indicates the total system mass.

\begin{tabular}{|c|c|}
\hline Parameter & Value \\
\hline$\Delta \alpha_{0}^{\star}$ (mas) & $152.30_{-1.37}^{+1.46}$ \\
\hline$\Delta \delta_{0}$ (mas) & $-203.80_{-0.86}^{+0.91}$ \\
\hline$\varpi_{\mathrm{abs}}$ (mas) & $28.57_{-0.61}^{+0.56}$ \\
\hline$\mu_{\alpha^{\star}}\left({\left.\operatorname{mas~} \mathrm{yr}^{-1}\right)}^{-1}\right.$ & $104.79_{-0.20}^{+0.20}$ \\
\hline$\mu_{\delta}\left({\left.\operatorname{mas~} \mathrm{yr}^{-1}\right)}^{-1}\right.$ & $-161.53_{-0.11}^{+0.11}$ \\
\hline$\rho$ (mas) & $-26.34_{-0.33}^{+0.35}$ \\
\hline$P(\mathrm{~d})$ & $690.68_{-3.59}^{+3.41}$ \\
\hline$P(\mathrm{yr})$ & $1.891_{-0.010}^{+0.009}$ \\
\hline$\Omega\left({ }^{\circ}\right)$ & $113.83_{-7.64}^{+7.43}$ \\
\hline$\lambda_{\text {ref }}\left({ }^{\circ}\right)$ & $-51.84_{-8.06}^{+8.31}$ \\
\hline$\sqrt{e} \sin \omega()$ & $-0.29_{-0.07}^{+0.09}$ \\
\hline$\sqrt{e} \cos \omega()$ & $-0.15_{-0.21}^{+0.32}$ \\
\hline$\sqrt{M_{2}} \sin i\left(M_{\mathrm{Jup}}\right)$ & $4.43_{-0.53}^{+0.45}$ \\
\hline$\sqrt{M_{2}} \cos i\left(M_{\mathrm{Jup}}\right)$ & $6.88_{-0.30}^{+0.23}$ \\
\hline$s_{\alpha}(\mathrm{mas})$ & $1.59_{-0.08}^{+0.09}$ \\
\hline$s_{\delta}(\mathrm{mas})$ & $1.24_{-0.06}^{+0.07}$ \\
\hline$e()$ & $0.146_{-0.053}^{+0.074}$ \\
\hline$\omega\left(^{\circ}\right)$ & $-114.09_{-29.14}^{+59.26}$ \\
\hline$i\left(^{\circ}\right)$ & $32.90_{-3.44}^{+2.94}$ \\
\hline$T_{P}(\mathrm{~d})$ & $57502.49_{-52.28}^{+110.81}$ \\
\hline$\alpha$ (mas) & $10.00_{-0.26}^{+0.29}$ \\
\hline$a_{1}$ (mas) & $10.22_{-0.26}^{+0.30}$ \\
\hline$a_{\text {rel }}$ (mas) & $22.90_{-0.69}^{+0.44}$ \\
\hline$a_{\mathrm{rel}}(\mathrm{au})$ & $0.80_{-0.02}^{+0.01}$ \\
\hline$M_{2}\left(M_{\text {Jup }}\right)$ & $66.95_{-4.84}^{+4.41}$ \\
\hline$M_{2}\left(M_{\text {Sun }}\right)$ & $0.064_{-0.005}^{+0.004}$ \\
\hline$M_{\mathrm{tot}}\left(M_{\mathrm{Sun}}\right)$ & $0.145_{-0.011}^{+0.005}$ \\
\hline \multicolumn{2}{|c|}{ Priors } \\
\hline$M_{1}\left(M_{\text {Sun }}\right)$ & $0.081_{-0.008}^{+0.002}$ \\
\hline$\Delta \varpi$ (mas) & $0.82_{-0.11}^{+0.11}$ \\
\hline$\Delta \mathrm{mag}$ & $5.08_{-0.32}^{+0.33}$ \\
\hline
\end{tabular}

(2016b). We used the emcee package (Foreman-Mackey et al. 2013) to implement the MCMC and expressed the binary model using pystrometry (Sahlmann 2019b) ${ }^{8}$ with the parameter vector $\boldsymbol{\theta}$ composed of $\Delta \alpha_{0}^{\star}, \Delta \delta_{0}, \varpi, \mu_{\alpha^{\star}}, \mu_{\delta}, \rho, P, \sqrt{e} \sin \omega$, $\sqrt{e} \cos \omega, \lambda_{\text {Ref }}, s_{\alpha}, s_{\delta}, M_{2} \sin i, M_{2} \cos i$, and $\Omega$. We added the systemic velocity $\gamma$ when RV data were included. The magnitude difference $\Delta$ mag and the parallax correction $\Delta \varpi$ are incorporated as Gaussian priors in the MCMC (see Sahlmann et al. 2016b), whereas the primary mass $M_{1}$ prior was implemented as described in the previous section. Finally, the reference time $T_{\text {Ref }}$ and the absolute coordinates enter the model as constants.

\footnotetext{
${ }^{8}$ https://github.com/Johannes-Sahlmann/pystrometry
} 

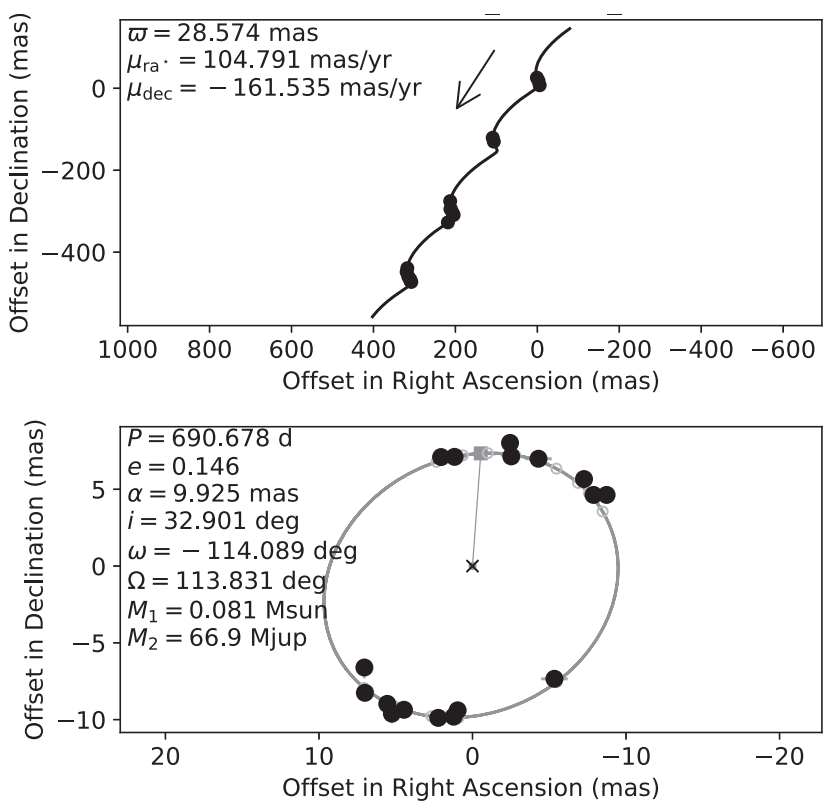

Figure 7. Top: Sky-projected motion of 2M1059-21 measured with GMOS-S after removal of orbital motion and chromatic refraction. Bottom: Sky-projected photocentre orbit. The cross identifies the barycentre and the grey square shows the periastron location. Epoch-averaged measurements are shown with black circles and the 'median model' is shown by the curve. The individual frame measurement that are actually used in the analysis are not shown for clarity.
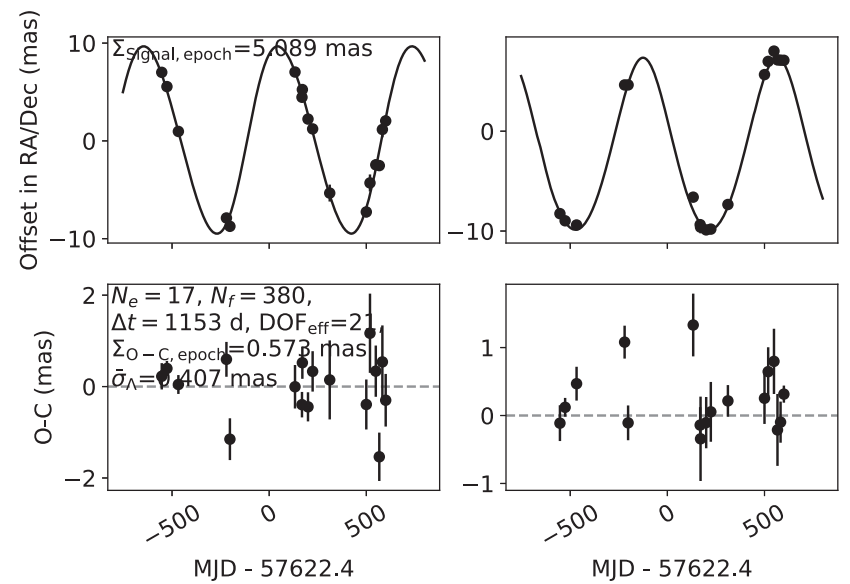

MJD - 57622.4

Figure 8. The top panels show the orbital motion of 2M1059-21 in RA (left) and Dec. (right) as a function of time with the epoch-averaged residuals at the bottom. Individual-frame measurements are not shown for clarity.

\section{RESULTS}

\subsection{The orbit of 2M1059-21}

The Genetic Algorithm identified a unique solution that corresponds to a low-eccentricity orbit with a period of $\sim 690 \mathrm{~d}$. The subsequent MCMC analysis provided well-constrained parameters with fast chain convergence and small parameter correlations. Table 6 lists the adopted solution parameters determined as the median of the posterior distributions with $1 \sigma$ equivalent confidence intervals. Figs 7 and 8 show the measured astrometric motion, the fitted Keplerian orbit, and the residuals of the model. The predicted RV orbit is shown in Fig. 9 together with the two available RV

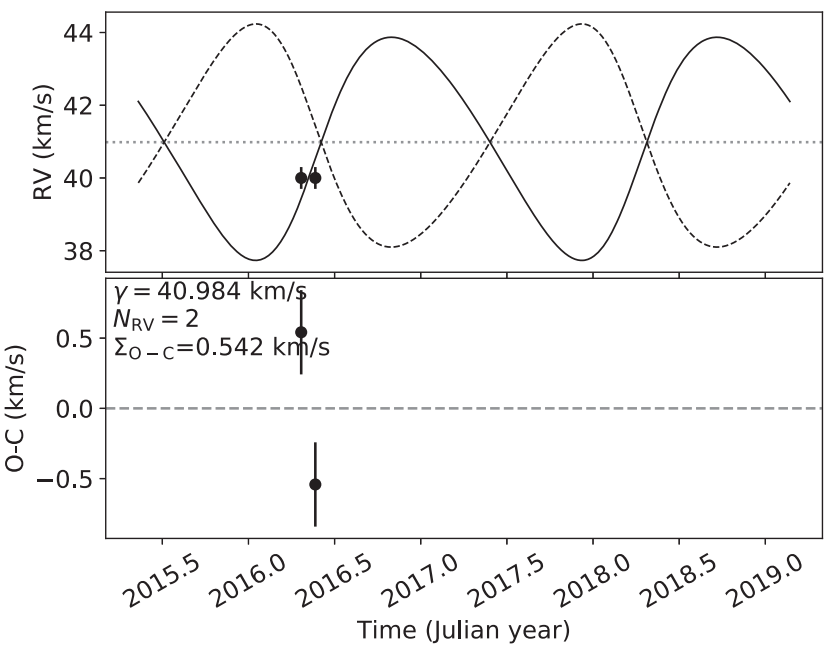

Figure 9. Top: The predicted radial velocity curve of 2M1059-21 according to our solution (solid line) and the degenerate solution with $\omega+180^{\circ}$ and $\Omega+180^{\circ}$ (dashed line). Bottom: The residual after fitting for the systemic velocity as single parameter while keeping all other parameters fixed.

measurements (Table 2), which allowed us to determine a systemic velocity of $\gamma=40.98 \pm 0.54 \mathrm{~km} \mathrm{~s}^{-1}$. We cannot use these RVs to distinguish between the nominal and degenerate solution, and for the degenerate orbit with $\omega+180^{\circ}$ and $\Omega+180^{\circ}$ we determine an alternative systemic velocity of $\gamma^{\prime}=39.06 \pm 0.54 \mathrm{~km} \mathrm{~s}^{-1}$.

Our results unambiguously confirm the binary nature of 2M1059-21 and determine its orbital solution for the first time. The measured absolute parallax places the system at a distance of $35 \mathrm{pc}$. The orbit is moderately eccentric $(e \simeq 0.14)$ and the 1.9yr period corresponds to a relative semimajor axis of 0.8 au. For a primary mass of $0.082_{-0.009}^{+0.002} \mathrm{M}_{\odot}$, we determine the mass of the T3-dwarf companion at $0.064_{-0.005}^{+0.004} \mathrm{M}_{\odot}$.

\subsection{The orbit of $2 \mathrm{M} 0805+48$}

The RV orbit of $2 \mathrm{M} 0805+48$ was previously determined by Burgasser et al. (2016) to have an orbital period $2.02 \pm 0.03 \mathrm{yr}$ and eccentricity of $0.46 \pm 0.05$. Even when left unconstrained by radial motion, the Genetic Algorithm applied to our astrometry of $2 \mathrm{M} 0805+48$ identified a unique solution with a well-matched orbital period and eccentricity.

The MCMC for 2M0805+48 was implemented with a simultaneous fit to both astrometry and RV data, where we used the three additional RV measurements listed in Table 3 along with values from Burgasser et al. (2016a).

Again, this analysis revealed well-constrained parameters with fast chain convergence and small parameter correlations. Table 7 lists the adopted solution parameters. Figs 10 and 11 show the measured astrometric motion, the fitted Keplerian orbit, and the residuals of the model. The RV orbit is shown in Fig. 12. The direct use of RV data allowed us to break the degeneracy in $\omega$ and $\Omega$; hence, this is the unique solution.

We confirm the period and eccentricity determinations of Burgasser et al. (2016a) and the incorporation of astrometric and addition RV data leads to much tighter constraints on these parameters. By determining the astrometric orbit of $2 \mathrm{M} 0805+48$ for the first time, we also confirm their prediction of a nearly edge-on orbit with an inclination of $112 \pm 2^{\circ}$. 
Table 7. MCMC posterior parameters of $2 \mathrm{M} 0805+48$.

\begin{tabular}{|c|c|}
\hline Parameter & Value \\
\hline$\Delta \alpha_{0}^{\star}$ (mas) & $-293.19_{-0.41}^{+0.42}$ \\
\hline$\Delta \delta_{0}(\mathrm{mas})$ & $-5.05_{-0.78}^{+0.78}$ \\
\hline$\varpi_{\mathrm{abs}}(\mathrm{mas})$ & $39.91_{-0.34}^{+0.35}$ \\
\hline$\mu_{\alpha^{\star}}\left(\operatorname{mas~yr}^{-1}\right)$ & $-443.76_{-0.28}^{+0.29}$ \\
\hline$\mu_{\delta}\left(\operatorname{mas~yr}^{-1}\right)$ & $48.82_{-0.32}^{+0.31}$ \\
\hline$\rho$ (mas) & $35.06_{-0.64}^{+0.61}$ \\
\hline$\gamma\left(\mathrm{m} \mathrm{s}^{-1}\right)$ & $10574.07_{-140.20}^{+135.69}$ \\
\hline$P(\mathrm{~d})$ & $740.43_{-1.63}^{+1.57}$ \\
\hline$P(\mathrm{yr})$ & $2.027_{-0.004}^{+0.004}$ \\
\hline$\Omega\left({ }^{\circ}\right)$ & $-13.69_{-2.00}^{+2.02}$ \\
\hline$\lambda_{\text {ref }}\left({ }^{\circ}\right)$ & $-294.65_{-1.71}^{+1.82}$ \\
\hline$\sqrt{e} \sin \omega()$ & $-0.54_{-0.04}^{+0.04}$ \\
\hline$\sqrt{e} \cos \omega()$ & $0.37_{-0.05}^{+0.05}$ \\
\hline$\sqrt{M_{2}} \sin i\left(M_{\text {Jup }}\right)$ & $7.54_{-0.83}^{+0.32}$ \\
\hline$\sqrt{M_{2}} \cos i\left(M_{\mathrm{Jup}}\right)$ & $-2.98_{-0.27}^{+0.36}$ \\
\hline$s_{\alpha}(\mathrm{mas})$ & $1.82_{-0.11}^{+0.13}$ \\
\hline$s_{\delta}(\mathrm{mas})$ & $1.92_{-0.12}^{+0.13}$ \\
\hline$e$ & $0.423_{-0.019}^{+0.019}$ \\
\hline$\omega\left(^{\circ}\right)$ & $-55.79_{-5.36}^{+5.36}$ \\
\hline$i\left(^{\circ}\right)$ & $111.85_{-1.52}^{+1.55}$ \\
\hline$T_{P}(\mathrm{~d})$ & $58840.28_{-9.19}^{+9.17}$ \\
\hline$\alpha$ (mas) & $14.76_{-0.38}^{+0.38}$ \\
\hline$a_{1}$ (mas) & $15.67_{-0.39}^{+0.39}$ \\
\hline$a_{\text {rel }}$ (mas) & $32.58_{-3.69}^{+1.01}$ \\
\hline$a_{\mathrm{rel}}(\mathrm{au})$ & $0.82_{-0.09}^{+0.02}$ \\
\hline$M_{2}\left(M_{\mathrm{Jup}}\right)$ & $66.28_{-14.04}^{+5.18}$ \\
\hline$M_{2}\left(M_{\text {Sun }}\right)$ & $0.063_{-0.013}^{+0.005}$ \\
\hline$M_{\mathrm{tot}}\left(M_{\mathrm{Sun}}\right)$ & $0.134_{-0.038}^{+0.011}$ \\
\hline \multicolumn{2}{|c|}{ Priors } \\
\hline$\Delta \mathrm{mag}$ & $3.81_{-0.07}^{+0.07}$ \\
\hline$M_{1}\left(M_{\text {Sun }}\right)$ & $0.069_{-0.027}^{+0.008}$ \\
\hline$\Delta \varpi(\mathrm{mas})$ & $0.54_{-0.11}^{+0.11}$ \\
\hline
\end{tabular}

\section{DISCUSSION}

\subsection{Masses of $T$ dwarf companions}

Our astrometric follow-up allowed us to set tight constraints on the masses of the T dwarf companions of 2M1059-21 and $2 \mathrm{M} 0805+48$. Table 8 summarizes those mass determinations. In the context of other dynamical T dwarf masses (e.g. table 6 of Dupuy et al. 2019), we see that the mass of $2 \mathrm{M} 0805+48 \mathrm{~B}$ is almost equal to the one of WI0720-08B estimated by Dupuy et al. (2019), which also has the same spectral type, yet a mass higher than the other three T5 dwarfs with measured dynamical masses. From this comparison, $2 \mathrm{M} 0805+48$ is also a 'massive' T dwarf.

Our mass of $67_{-5}^{+4} M_{\text {Jup }}$ for the T3.5 2M1059-21B is also significantly higher than its two spectral type equivalents DENIS J2252-1730B (T3.5, $41 \pm 4 M_{\text {Jup }}$ ) and 2MASS J1534-2952A (T4.5, $\left.51 \pm 5 M_{\text {Jup }}\right)$.
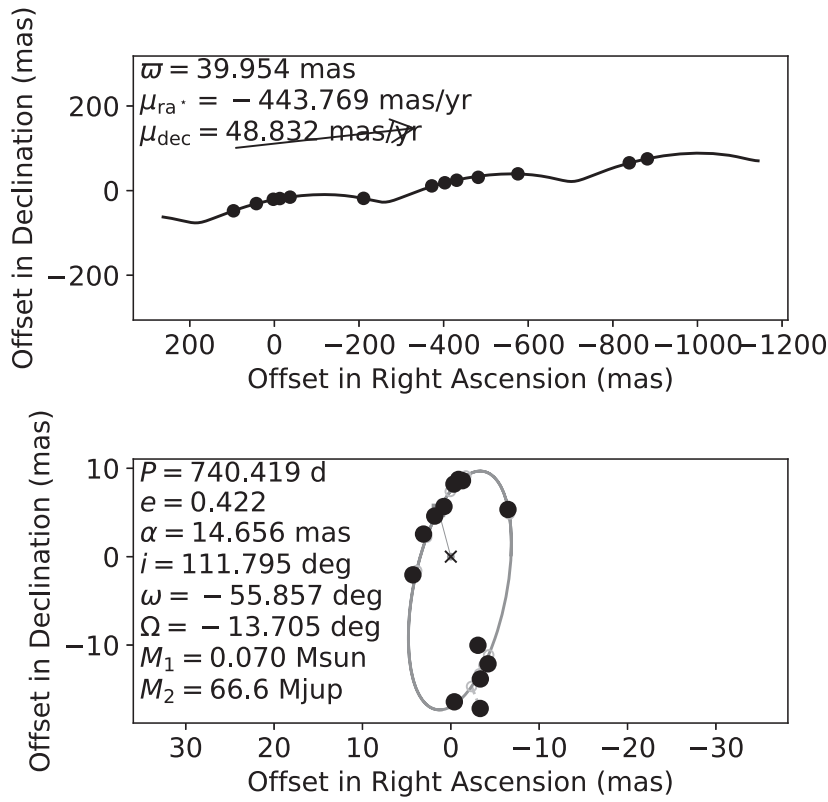

Figure 10. Sky-projected motion of $2 \mathrm{M} 0805+48$ measured with GMOSN, cf. Fig. 7.
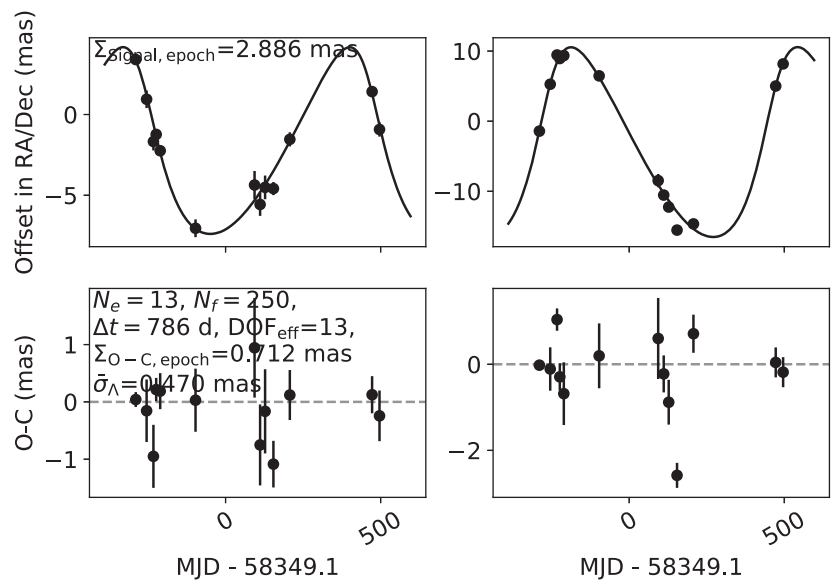

Figure 11. Orbital motion of $2 \mathrm{M} 0805+48$ as a function of time, cf. Fig. 8.

In Fig. 13, we show our results in the context of other low-mass systems with dynamically determined masses, where we included early-to-mid M dwarfs from Schweitzer et al. (2019) and lateM, L, and T dwarfs from Dupuy \& Liu (2017), Lazorenko \& Sahlmann (2018), and Dupuy et al. (2019). The curves correspond to isochrones of different ages $(0.3,0.5,1,5$, and $12 \mathrm{Gyr}$; Baraffe et al. 2015), where we used the spectral type - effective temperature calibrations of Houdebine et al. (2019) for M dwarfs and of Stephens et al. (2009) for L and T dwarfs to convert theoretically predicted effective temperatures into spectral types.

This figure shows that the results of this work are generally consistent with the dynamical masses from other groups in the spectral-type range probed and that $2 \mathrm{M} 0805+48 \mathrm{~B}$ is among the latest-type dwarfs with well-constrained masses. The masses derived for each member of our two pairs are compatible with the 5 and 12 Gyr isochrones at the $1 \sigma$ level and the $>1$ Gyr isochrones show reasonable agreement with the observational data. 


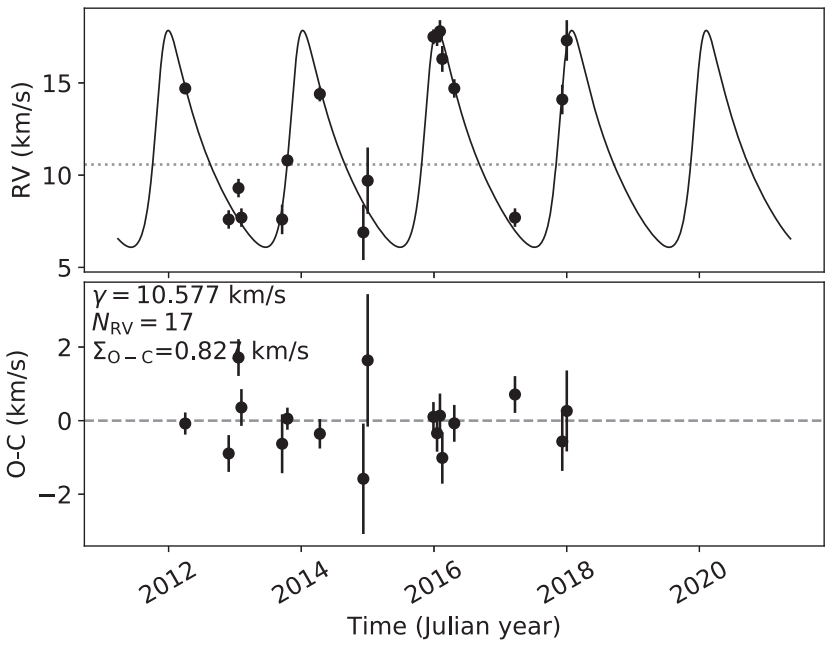

Figure 12. RV orbit of $2 \mathrm{M} 0805+48$. The curve in the top panel shows the model resulting from the simultaneous fit to astrometry and RV data. The bottom panel shows the residuals.

2M1059-21 and 2M0805+48 join a short yet growing sample of 'massive' $\mathrm{T}$ dwarfs compared with their predictions from evolutionary models, alongside $\epsilon$ Indi B and C (Dieterich et al. 2018), WI0720-08 (Dupuy et al. 2019), Gl 229 B (Brandt et al. 2019), and HD 4113C (Cheetham et al. 2018). Several avenues have been explored to interpret the high mass of Gl 229 B within the framework of current evolutionary models, including unresolved binarity, incorrect astrometry of the primary, low metallicity, and atypical old ages ( $\gtrsim 7 \mathrm{Gyr}$ ); however, none of these hypotheses have
Table 8. Dynamical masses of the two T-dwarf companions.

\begin{tabular}{lcc}
\hline Object & Spec. type & Mass \\
\hline 2M1059-21A & L0.5 & $0.081_{-0.008}^{+0.002} M_{\text {Sun }}$ \\
2M1059-21B & T3.5 & $0.064_{-0.005}^{+0.004} M_{\text {Sun }}$ \\
2M1059-21B & T3.5 & $67_{-5}^{+4} M_{\text {Jup }}$ \\
2M0805+48A & L4 & $0.069_{-0.027}^{+0.008} M_{\text {Sun }}$ \\
2M0805+48B & T5.5 & $0.063_{-0.013}^{+0.005} M_{\text {Sun }}$ \\
2M0805+48B & T5.5 & $66_{-14}^{+5} M_{\text {Jup }}$ \\
\hline
\end{tabular}

been determined as the cause. In particular, activity and kinematic constraints for the age of the primary lead to a broad range of 2-8 Gyr, but with a higher likelihood at younger ages.

The T dwarf secondaries of 2M1059-21 and 2M0805+48 also have dynamical masses higher than comparable objects at a given spectral type. These 'massive' $\mathrm{T}$ dwarfs are marginally consistent with older ages ( $\gtrsim 7 \mathrm{Gyr}$ ), yet do not show signatures of low metallicity. Recent theoretical and computational developments on the equation of state (EOS) describing the interiors of substellar objects, based on improved quantum molecular dynamics calculations in the high density-temperature regime of pressure dissociation and ionization for $\mathrm{H}$ and $\mathrm{He}$, can provide a partial solution to this discrepancy. The evolutionary models associated with this EOS lead to more degenerate interiors, slightly faster cooling rates, cooler temperatures, and lower brightness for a given mass (Phillips et al. 2020).
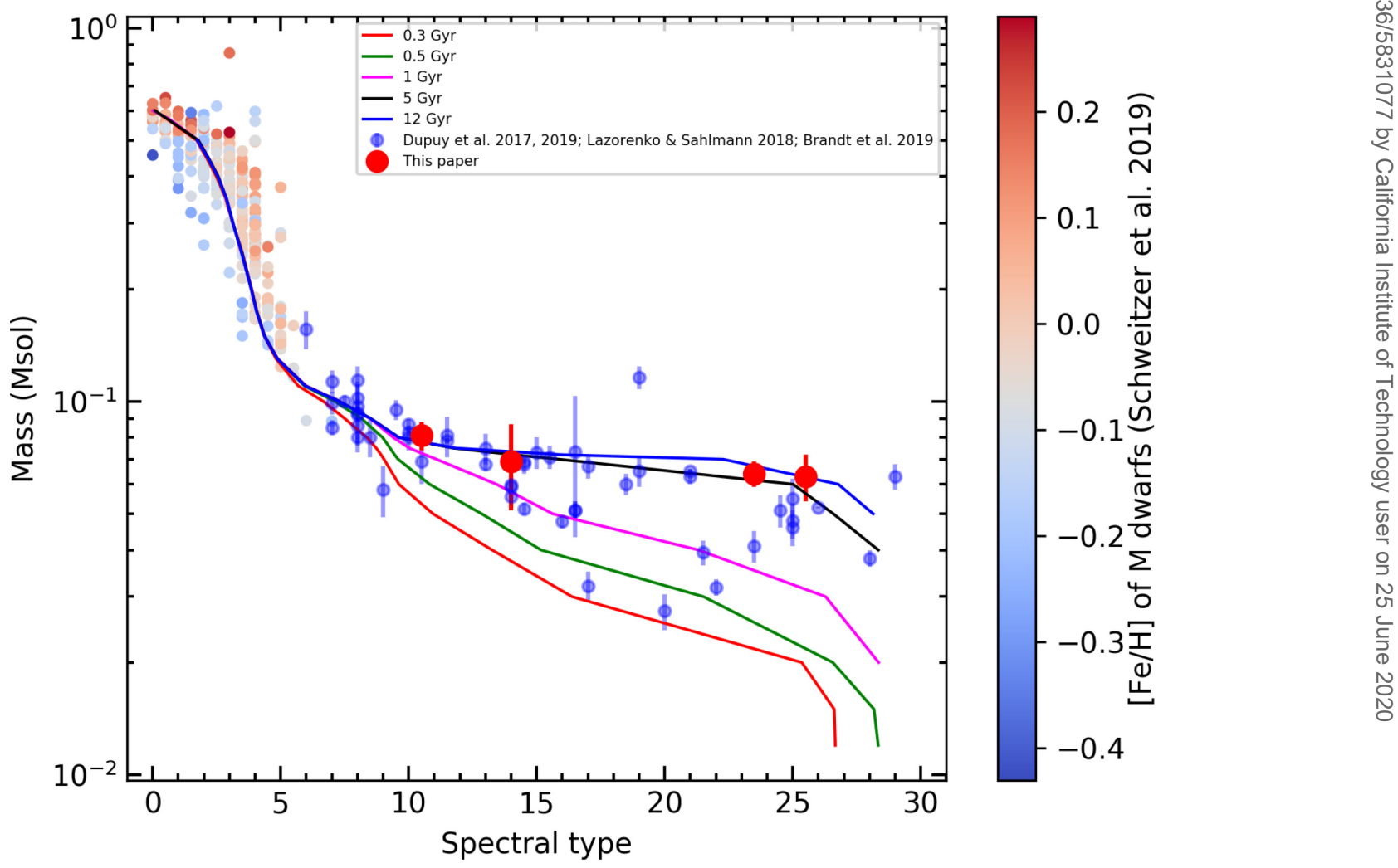

Figure 13. Dynamically derived masses in the low-luminosity regime as a function of spectral type. On the $x$-axis, spectral types of M0, L0, and T0 are encoded as 0,10 , and 20, respectively. The 2M0805+48 and 2M1059-21 systems are shown with large red symbols. 
Table 9. Absolute parallaxes and proper motions.

\begin{tabular}{lrr}
\hline Parameter & \multicolumn{1}{c}{ GMOS } & \multicolumn{1}{c}{ GDR2 } \\
\hline$\varpi_{\text {abs }}(\mathrm{mas})$ & $28.6 \pm 0.6$ & $31.4 \pm 0.8$ \\
$\mu_{\alpha^{\star}}\left(\mathrm{mas} \mathrm{yr}^{-1}\right)$ & $99.1 \pm 0.6$ & $85.0 \pm 1.2$ \\
$\mu_{\delta}\left(\mathrm{mas} \mathrm{yr}^{-1}\right)$ & $-161.7 \pm 0.4$ & $-164.2 \pm 1.0$ \\
$\varpi_{\text {abs }}\left(\mathrm{mas}^{2}\right)$ & $40.0 \pm 0.4$ & $46.8 \pm 1.0$ \\
$\mu_{\alpha^{\star}, \text { abs }}\left(\mathrm{mas} \mathrm{yr}^{-1}\right)$ & $-442.3 \pm 0.8$ & $-459.1 \pm 1.4$ \\
$\mu_{\delta, \text { abs }}\left(\mathrm{mas} \mathrm{yr}^{-1}\right)$ & $48.1 \pm 0.5$ & $56.7 \pm 1.1$ \\
\hline
\end{tabular}

\subsection{Astrometric accuracy achieved with GMOS imaging}

Using GMOS-S and GMOS-N imaging, we achieved epoch residual rms values of 0.6 and 0.7 mas for 2M1059-21 and $2 \mathrm{M} 0805+48$, respectively. We expect that improved source extraction procedures, better selection of the reference star sample, and more careful treatment of outliers during the iterative astrometry fitting will result in significantly better performance. However, we do not expect to reach the 0.1 mas accuracy performance of our FORS2/VLT programme (Sahlmann et al. 2014), because the GMOS fields have significantly fewer available reference stars.

\subsection{Comparison with Gaia DR2 parameters}

In Table 9, we compare our parallax and proper motion determinations with the values given in the GDR2 catalogue. In all cases, the differences are significant that can primarily be attributed to the GDR2 model that did not account for orbital motion but applied the standard five-parameter linear model instead. This is also reflected in the elevated GDR2 astrometric excess noise of 2.7 and 4.3 mas for 2M1059-21 and $2 \mathrm{M} 0805+48$, respectively. As a result the GDR2 parameters are biased and we expect out determinations to be more accurate. In particular, our parallax measurements imply significantly larger distances.

\section{CONCLUSIONS}

We reported the first results of an astrometric follow-up campaign to confirm and characterize spectral binary brown dwarfs. Using both Gemini GMOS imagers, we demonstrated sub-milliarcsecond astrometry and determined the astrometric orbits of 2M1059-21 and $2 \mathrm{M} 0805+48$ for the first time, thereby tightly constraining the masses of their T-dwarf companions. We showed that astrometric observations represent an underutilized avenue for confirming and characterizing the orbits of tight low-mass binaries containing stellar and brown dwarf components. Our survey is particularly efficient because it is guided by prior indications for binarity from near-infrared spectroscopy.

Surveys like ours combined with the ultracool-dwarf binary orbits expected from the Gaia mission and astrometric programmes that explore the presence of giant planets around ultracool dwarfs will lead to the population characterization of compact ultracool systems over a wide range of mass ratios.

\section{ACKNOWLEDGEMENTS}

This research made use of the data bases at the Centre de Données astronomiques de Strasbourg (http://cds.u-strasbg.fr); of NASA's Astrophysics Data System Service (http://adsabs.harvard .edu/abstract_service.html); of the paper repositories at arXiv; and of ASTROPY, a community-developed core PYTHON package for Astronomy (Astropy Collaboration et al. 2013).

Based on observations obtained at the Gemini Observatory (acquired through the Gemini Observatory Archive and processed using the Gemini IRAF package and gemini_python), which is operated by the Association of Universities for Research in Astronomy, Inc., under a cooperative agreement with the NSF on behalf of the Gemini partnership: the National Science Foundation (United States), the National Research Council (Canada), CONICYT (Chile), Ministerio de Ciencia, Tecnología e Innovación Productiva (Argentina), and Ministério da Ciência, Tecnologia e Inovação (Brazil).

This work has made use of data from the European Space Agency (ESA) mission Gaia (https://www.cosmos.esa.int/gaia), processed by the Gaia Data Processing and Analysis Consortium (DPAC; ht tps://www.cosmos.esa.int/web/gaia/dpac/consortium). Funding for the DPAC has been provided by national institutions, in particular the institutions participating in the Gaia Multilateral Agreement.

ELM acknowledges funding from the Fondo Europeo de Desarrollo Regional (MINECO/FEDER) under grant no. AYA201569350-C3-1-P. DBG and AJB acknowledge funding support from the National Aeronautics and Space Administration under Grant No. NNX15AI75G.

\section{REFERENCES}

Allen P. R., Koerner D. W., Reid I. N., Trilling D. E., 2005, ApJ, 625, 385 Allers K. N. et al., 2007, ApJ, 657, 511

Astropy Collaboration et al., 2013, A\&A, 558, A33

Baraffe I., Homeier D., Allard F., Chabrier G., 2015, A\&A, 577, A42

Bardalez Gagliuffi D. C. et al., 2014, ApJ, 794

Bardalez Gagliuffi D. C., Gelino C. R., Burgasser A. J., 2015, AJ, 150, 163

Basri G., Martín E. L., 1999, AJ, 118, 2460

Bennett D. P. et al., 2008, ApJ, 684, 663

Berger E., 2006, ApJ, 648, 629

Bertin E., 2006, in Gabriel C., Arviset C., Ponz D., Enrique S., eds, ASP Conf. Ser. Vol. 351, Astronomical Data Analysis Software and Systems

XV. Astron. Soc. Pac., San Francisco, p. 112

Bertin E., Arnouts S., 1996, A\&AS, 117, 393

Blake C. H., Charbonneau D., White R. J., Torres G., Marley M. S., Saumon D., 2008, ApJ, 678, L125

Blake C. H., Charbonneau D., White R. J., 2010, ApJ, 723, 684

Brandner W., Martín E. L., Bouy H., Köhler R., Delfosse X., Basri G., Andersen M., 2004, A\&A, 428, 205

Brandt T. D., Dupuy T. J., Bowler B. P., Bardalez Gagliuffi D. C., Faherty J., Mirek Brandt G., Michalik D., 2019, preprint (arXiv:1910.01652)

Burgasser A. J., 2004, ApJS, 155, 191

Burgasser A. J., 2007, AJ, 134, 1330

Burgasser A. J., Blake C. H., 2009, AJ, 137, 4621

Burgasser A. J., Liu M. C., Ireland M. J., Cruz K. L., Dupuy T. J., 2008, ApJ, 681, 579

Burgasser A. J., Cruz K. L., Cushing M., Gelino C. R., Looper D. L., Faherty J. K., Kirkpatrick J. D., Reid I. N., 2010, ApJ, 710, 1142

Burgasser A. J., Gelino C. R., Cushing M. C., Kirkpatrick J. D., 2012a, ApJ, 745,26

Burgasser A. J., Luk C., Dhital S., Bardalez Gagliuffi D., Nicholls C. P., Prato L., West A. A., Lépine S., 2012b, ApJ, 757, 110

Burgasser A. J. et al., 2015a, AJ, 149, 104

Burgasser A. J., Melis C., Todd J., Gelino C. R., Hallinan G., Bardalez Gagliuffi D., 2015b, AJ, 150, 180

Burgasser A. J., Blake C. H., Gelino C. R., Sahlmann J., Bardalez Gagliuffi D., 2016, ApJ, 827, 25

Cepa J. et al., 2000, SPIE Conf. Ser. Vol. 4008, Optical and IR Instrumentation and Detectors, p. 623

Cheetham A. et al., 2018, A\&A, 614, A16 
Cruz K. L., Reid I. N., Liebert J., Kirkpatrick J. D., Lowrance P. J., 2003, AJ, 126, 2421

Cruz K. L., Burgasser A. J., Reid I. N., Liebert J., 2004, ApJ, 604, L61

Cruz K. L., Kirkpatrick J. D., Burgasser A. J., 2009, AJ, 137, 3345

Dahn C. C. et al., 2008, ApJ, 686, 548

Dieterich S. B. et al., 2018, ApJ, 865, 28

Dupuy T. J., Liu M. C., 2012, ApJS, 201, 19

Dupuy T. J., Liu M. C., 2017, ApJS, 231, 15

Dupuy T. J., Liu M. C., Ireland M. J., 2014, ApJ, 790, 133

Dupuy T. J. et al., 2019, AJ, 158, 174

Foreman-Mackey D., Hogg D. W., Lang D., Goodman J., 2013, PASP, 125, 306

Gaia Collaboration et al., 2016, A\&A, 595, A1

Gaia Collaboration, 2018 et al., A\&A, 616, A1

Gelino C. R., Burgasser A. J., 2010, AJ, 140, 110

Gimeno G. et al., 2016, SPIE Conf. Ser. Vol. 9908, Ground-based and Airborne Instrumentation for Astronomy VI, p. 2S

Guenther E. W., Wuchterl G., 2003, A\&A, 401, 677

Han C. et al., 2013, ApJ, 778, 38

Hawley S. L. et al., 2002, AJ, 123, 3409

Hayashi C., Nakano T., 1963, Prog. Theor. Phys., 30, 460

Hook I. M., Jørgensen I., Allington-Smith J. R., Davies R. L., Metcalfe N., Murowinski R. G., Crampton D., 2004, PASP, 116, 425

Houdebine É. R., Mullan D. J., Doyle J. G., de La Vieuville G., Butler C. J., Paletou F., 2019, AJ, 158, 56

Joergens V., Müller A., 2007, ApJ, 666, L113

Joergens V., Müller A., Reffert S., 2010, A\&A, 521, A24

Kirkpatrick J. D., 2005, ARA\&A, 43, 195

Kirkpatrick J. D. et al., 2008, ApJ, 689, 1295

Knapp G. R. et al., 2004, AJ, 127, 3553

Konopacky Q. M., Ghez A. M., Barman T. S., Rice E. L., Bailey J. I., White R. J., McLean I. S., Duchêne G., 2010, ApJ, 711, 1087

Koren S. C., Blake C. H., Dahn C. C., Harris H. C., 2016, AJ, 151, 57

Kumar S. S., 1963, ApJ, 137, 1121

Lazorenko P. F., 2006, A\&A, 449, 1271

Lazorenko P. F., Lazorenko G. A., 2004, A\&A, 427, 1127

Lazorenko P. F., Sahlmann J., 2018, A\&A, 618, A111

Lazorenko P. F., Mayor M., Dominik M., Pepe F., Ségransan D., Udry S., 2009, A\&A, 505, 903

Lazorenko P. F. et al., 2011, A\&A, 527, A25+
Lazorenko P. F., Sahlmann J., Ségransan D., Martín E. L., Mayor M., Queloz D., Udry S., 2014, A\&A, 565, A21

Magazzu A., Martin E. L., Rebolo R., 1993, ApJ, 404, L17

Martín E. L., Barrado y Navascués D., Baraffe I., Bouy H., Dahm S., 2003, ApJ, 594, 525

Martín E. L. et al., 2010, A\&A, 517, A53

Martin E. C. et al., 2017, ApJ, 838, 73

Martín E. L., Lodieu N., Pavlenko Y., Béjar V. J. S., 2018, ApJ, 856, 40

McLean I. S. et al., 1998, in Fowler A. M., ed., SPIE Conf. Ser. Vol. 3354, Optical and IR Instrumentation and Detectors. SPIE, Bellingham, p. 566

Miyazaki S. et al., 2018, AJ, 156, 136

Mohanty S., Basri G., 2003, ApJ, 583, 451

Pecaut M. J., Mamajek E. E., 2013, ApJS, 208, 9

Phillips M. W. et al., 2020, A\&A, 637, A38

Poleski R. et al., 2017, A\&A, 604, A103

Rebolo R., Zapatero Osorio M. R., Martín E. L., 1995, Nature, 377, 129

Reiners A., Basri G., 2008, ApJ, 684, 1390

Russell H. N., 1931, MNRAS, 91, 951

Sahlmann J., 2012, PhD thesis, Observatoire de Genève, Université de Genève

Sahlmann J., 2019a, Johannes-Sahlmann/gemini-reduction: First release

Sahlmann J., 2019b, Johannes-Sahlmann/pystrometry

Sahlmann J., Lazorenko P. F., Ségransan D., Martín E. L., Queloz D., Mayor M., Udry S., 2013, A\&A, 556, A133

Sahlmann J., Lazorenko P. F., Ségransan D., Martín E. L., Mayor M., Queloz D., Udry S., 2014, A\&A, 565, A20

Sahlmann J., Lazorenko P. F., Ségransan D., Martín E. L., Mayor M., Queloz D., Udry S., 2015, A\&A, 577, A15

Sahlmann J., Lazorenko P. F., Bouy H., Martín E. L., Queloz D., Ségransan D., Zapatero Osorio M. R., 2016a, MNRAS, 455, 357

Sahlmann J. et al., 2016b, A\&A, 595, A77

Sahlmann J., Lim P. L., Noss J., 2019, spacetelescope/pystortion: Release for Zenodo

Schweitzer A. et al., 2019, A\&A, 625, A68

Skrzypek N., Warren S. J., Faherty J. K., 2016, A\&A, 589, A49

Stephens D. C. et al., 2009, ApJ, 702, 154

Vogt H., 1926, Astron. Nachr., 226, 301

This paper has been typeset from a $\mathrm{T}_{\mathrm{E}} \mathrm{X} / \mathrm{L} \mathrm{T} \mathrm{E} \mathrm{X}$ file prepared by the author. 